\title{
Axisymmetric instabilities in gravitating discs with mass spectrum
}

\author{
V. G. Ortega ${ }^{1}$, E. Volkov ${ }^{2,1}$, and I. Monte-Lima ${ }^{1}$ \\ 1 Observatorio Nacional, DAGE, Rua Gen. Jose Cristino 77, Sao Cristovao, CEP 20921-400, Rio de Janeiro, \\ RJ, Brazil \\ 2 Sobolev Astronomical Institute, St. Petersburg State University, Russia
}

Received 21 February 2000 / Accepted 3 October 2000

\begin{abstract}
Starting from an axisymmetric, differentially rotating thin disc at equilibrium, in which the velocity dispersion is not large and in which the particles are characterized by a mass distribution and by a relationship between mass and velocity dispersion, we consider the stability of such a configuration against small axisymmetric disturbances. The analysis is performed kinetically and the behaviour of the marginal stability curves for different values of the system's parameters is investigated. Specifically, we consider the implications of changes in the parameters characterizing the mass spectrum range and the law relating the mass and velocity dispersion. Our results show that the characteristics of the system can significantly affect the critical stability parameters. We point out that such effects may strongly influence the evolution of the interstellar medium in disc galaxies.
\end{abstract}

Key words. ISM: kinematics and dynamics - ISM: clouds - ISM: molecules

\section{Introduction}

The work by Grishchuk \& Zel'dovich (1981), Polyachenko \& Fridman (1981) and Jog \& Solomon (1984) (see also Jog 1996) on the stability of multicomponent media has shown that the existence in a physical system of components with different random velocities is capable of modifying significantly the critical parameters and therefore the stability of such a system. Using the hydrodynamical approximation, Volkov \& Ortega (2000) analysed the conditions for the onset of gravitational instability in an ensemble of particles characterized by different random velocities anticorrelated with the masses of its constituents. They showed that for isotropic non-rotating and differentially rotating systems with a mass spectrum, the critical parameters, i.e. Jeans' length and Toomre's stability parameter, strongly depend on the width of the mass interval describing the particle distribution and also on the parameters of the mass-velocity dependence.

In the present paper, we employ the kinetic approach to investigate the stability of a flat, thin, differentially rotating disc configuration against local radial disturbances. We obtain the dispersion equation for such disturbances and compute the curves of marginal stability. These curves, calculated for several values of the parameters characterizing the mass spectrum and the particle

Send offprint requests to: V. G. Ortega, e-mail: vladimir@on.br dynamics, are compared with Toomre's (1964) well known curves. The behaviour of Toomre's stability parameter under various conditions and the possible role of certain instability conditions on the gas distributions observed in spiral galaxies are also considered.

\section{The disc configuration}

We consider a differentially rotating thin disc with an undisturbed distribution function of the Schwarzschild type:

$f_{0}=A m^{-\delta} \exp \left(-V^{2} / 2\right)$

where $A$ is a normalization constant, $\delta$ is a constant, $m$ is the mass and the quantity $V$ is given by

$V^{2}=\frac{v_{\mathrm{r}}^{2}}{\sigma_{\mathrm{r}}^{2}}+\frac{v_{\varphi}^{2}}{\sigma_{\varphi}^{2}}$.

Here $v_{\mathrm{r}}$ is the radial component and $v_{\varphi}$ the azimuthal component of the random velocity with dispersions $\sigma_{\mathrm{r}}$ and $\sigma_{\varphi}$ along those directions.

In the epicyclic approximation the components $\sigma_{\mathrm{r}}$ and $\sigma_{\varphi}$ of the velocity dispersion obey the relation

$\sigma_{\mathrm{r}}=\left(\frac{2 \Omega_{0}}{\kappa}\right) \sigma_{\varphi}$, 
where $\kappa$ is the epicyclic frequency and $\Omega_{0}$ the angular velocity determined by the unperturbed potential $\Phi_{0}$ through the equation

$r \Omega_{0}^{2}=\partial \Phi_{0} / \partial r$.

The angular velocity and its derivative at radius $r$ determines the epicyclic frequency at that position

$\kappa(r)=2 \Omega_{0}\left(1+\frac{r}{2 \Omega_{0}} \frac{\mathrm{d} \Omega_{0}}{\mathrm{~d} r}\right)^{1 / 2}$

The mass spectrum is considered to be contained within the interval $\left[m_{1}, m_{\mathrm{u}}\right], m_{\mathrm{l}}$ and $m_{\mathrm{u}}$ being the masses of the less and most massive elements respectively. We shall assume a power-law relationship between the velocity dispersion and the mass

$\sigma_{\mathrm{r}}(m)=\sigma_{1}\left(\frac{m}{m_{1}}\right)^{-\beta}$

where $\sigma_{1}$ is the radial velocity dispersion of the less massive elements. Introducing the dimensionless quantities $\xi$, $\eta$ given by $v_{\mathrm{r}}=V_{1} \xi, v_{\varphi}=V_{2} \eta$ with $V_{1}$ and $V_{2}$ defined by $V_{1}=\left(\frac{2 \Omega_{0}}{\kappa}\right) r \Omega_{0}, V_{2}=r \Omega_{0}$, the unperturbed distribution function may be written as

$f_{0}=A m^{-\delta} \exp \left[-\frac{V_{1}^{2}}{2 \sigma_{\mathrm{r}}^{2}}\left(\xi^{2}+\eta^{2}\right)\right]$.

Given such a system we wish to find its response to small perturbations of the undisturbed potential $\Phi_{0}$, considered to be axisymmetric. It is well known (Lin et al. 1969) that an asymptotic (WKBJ) solution of the linearized collisionless Boltzmann equation for disturbances of the type

$\Phi_{1} \propto \exp [i(\omega t-n \varphi+\chi)]$,

where $\omega$ is the radian frequency, $n$ is an integer $(n \geq 0)$ and $\chi$ is the radial phase, can be obtained in the form

$f_{1}=\frac{2 \Phi_{1}}{V_{1}^{2}} \frac{\mathrm{d} f_{0}}{\mathrm{~d} \tau^{2}}(1-q)$.

Here $f_{1}$ is the perturbed distribution function, $\tau^{2}=\xi^{2}+\eta^{2}$ and $q$ is given by the expression

$q=\frac{\nu}{2 \sin (\nu \pi)} \int_{-\pi}^{\pi} \mathrm{e}^{i[\nu s-\theta \xi \sin s+\theta \eta(1+\cos s)]} \mathrm{d} s$.

The quantities $\nu$ and $\theta$ defined as $\nu=\left(\omega-n \Omega_{0}\right) / \kappa$ and $\theta=(k r) \frac{2 \Omega_{0}^{2}}{\kappa^{2}}$, where $k$ is the radial wave vector, are dimensionless. Taking into account that

$\frac{\mathrm{d} f_{0}}{\mathrm{~d} \tau^{2}}=-\frac{V_{1}^{2}}{2 \sigma_{1}^{2}}\left(\frac{m}{m_{1}}\right)^{2 \beta} f_{0}$

and using (2) it follows that

$f_{1}=-\frac{\Phi_{1}}{\sigma_{1}^{2}}\left(\frac{m}{m_{1}}\right)^{2 \beta} f_{0}(1-q)$.

Defining further the surface density

$\mu=\int_{m_{1}}^{m_{\mathrm{u}}} \int_{-\infty}^{\infty} \int_{-\infty}^{\infty} m f \mathrm{~d} m \mathrm{~d} \xi \mathrm{d} \eta$ it can be shown that the perturbed $\mu_{1}$ and undisturbed $\mu_{0}$ densities are related by

$\frac{\mu_{1}}{\mu_{0}}=2 \pi G \Phi_{1} \frac{(2-\delta-2 \beta)\left(M^{2-\delta}-1\right)}{(2-\delta)\left(M^{2-\delta-2 \beta}-1\right)} \frac{k}{\kappa^{2}} \frac{F_{\nu}(p)}{\left(1-\nu^{2}\right)}$,

where $M=m_{\mathrm{u}} / m_{1}, p=k^{2} \sigma_{1}^{2} / \kappa^{2}$ and $F_{\nu}(p)$ is a function which we define below. Inserting in the last equation the potential $\Phi_{1}=-2 \pi G \mu_{1} / k$ obtained from Poisson's equation we get the dispersion relation

$\frac{k_{\mathrm{T}}}{k}\left(1-\nu^{2}\right)=\gamma F_{\nu}(p)$

where $k_{\mathrm{T}}=\kappa^{2} / 2 \pi G \mu_{0}$ is Toomre's wave number and $\gamma$ is given by

$\gamma=\frac{(2-\delta-2 \beta)\left(M^{2-\delta}-1\right)}{(2-\delta)\left(M^{2-\delta-2 \beta}-1\right)}$

The function $F_{\nu}(p)$ entering the dispersion equation depends on $\delta, \beta$ and $M$ characterizing the mass spectrum. It has the following form

$F_{\nu}(p)=\frac{1-\nu^{2}}{p}\left[1-\frac{\nu}{2 \sin (\nu \pi)} \int_{-\pi}^{\pi} \cos (\nu s) D_{\mathrm{s}}(p) \mathrm{d} s\right]$,

where

$D_{\mathrm{S}}(p)=\frac{(2-\delta)}{\left(M^{2-\delta}-1\right)} \int_{1}^{M} x^{1-\delta} \mathrm{e}^{-p(1+\cos s) / x^{2 \beta}} \mathrm{d} x$.

Equation (10) describes the response of the system to short-wave disturbances of the type given by formula (7). The particular case of radial, time-independent disturbances investigated by Toomre (1964) results from (10) by making $\nu \rightarrow 0, \omega \rightarrow 0$. The equation for marginal stability may then be written as

$\frac{k \sigma_{1}^{2}}{2 \pi G \mu_{0} \gamma}=1-\frac{2-\delta}{M^{2-\delta}-1} \int_{1}^{M} x^{1-\delta} I_{0}\left(\frac{p}{x^{2 \beta}}\right) \mathrm{e}^{-\frac{p}{x^{2 \beta}}} \mathrm{d} x$,

where $I_{0}$ is the modified Bessel function.

For the limiting case of a cold disc $(p \rightarrow 0)$, this equation gives, as expected, the Toomre critical wavenumber $k=k_{\mathrm{T}}$. For the limiting case of a rotationless hot disc $(p \rightarrow \infty)$, we obtain $k=2 \pi G \mu_{0} \gamma / \sigma_{1}^{2}$. The velocity dispersion $\sigma_{l}$ may be expressed in terms of the average radial velocity dispersion $\sigma_{0 \mathrm{r}}$. To do this, we fix the density, $\mu_{0}$, and the total specific random energy, $\sigma_{\mathrm{T}}$,

$\sigma_{\mathrm{T}}^{2}=\frac{1}{\mu_{0}} \int_{m_{1}}^{m_{\mathrm{u}}} m \psi(m) \sigma^{2}(m) \mathrm{d} m$,

where $\sigma(m)$ is the velocity dispersion of particles of mass $m$ and $\psi(m)$ the mass distribution function

$\psi(m)=\int_{-\infty}^{\infty} \int_{-\infty}^{\infty} f_{0} \mathrm{~d} \xi \mathrm{d} \eta$.

Using (3) and (8) we find

$\psi(m)=\frac{2 \pi \sigma_{1}^{2}}{V_{1}^{2}} A m_{1}^{(2 \beta-\alpha)}\left(\frac{m}{m_{1}}\right)^{-\alpha}$ 
and

$\mu_{0}=\frac{2 \pi \sigma_{1}^{2}}{V_{1}^{2}} A m_{1}^{(2-\alpha+2 \beta)}\left(\frac{M^{2-\alpha}-1}{2-\alpha}\right)(\alpha \neq 2)$.

Here $\alpha=\delta+2 \beta$ is the spectral index of the mass distribution. Thus, the value of $\delta$ is known once $\alpha$ and $\beta$ are given. When $\alpha=2$ the last formula becomes

$\mu_{0}=\frac{2 \pi \sigma_{1}^{2}}{V_{1}^{2}} A m_{1}^{(2 \beta-\alpha)} \ln (M)$.

In the epicyclic approximation

$\sigma^{2}(m)=\sigma_{\mathrm{r}}^{2}(m)\left[1+\left(\frac{\kappa}{2 \Omega_{0}}\right)^{2}\right]$,

so that from Eq. (15) we have

$\frac{\sigma_{0 \mathrm{r}}^{2}}{\sigma_{\mathrm{l}}^{2}}=\frac{1}{\mu_{0}} \int_{m_{1}}^{m_{\mathrm{u}}} m \psi(m)\left(\frac{m}{m_{1}}\right)^{-2 \beta} \mathrm{d} m$,

where

$\sigma_{0 \mathrm{r}}^{2}=\frac{\sigma_{\mathrm{T}}^{2}}{1+\left(\frac{\kappa}{2 \Omega_{0}}\right)^{2}}$.

Making use of the mass distribution given by (16) and the expression for the density $\mu_{0}$ we finally obtain

$\frac{\sigma_{0 \mathrm{r}}^{2}}{\sigma_{\mathrm{l}}^{2}}=\frac{(2-\alpha)\left(M^{2-2 \beta-\alpha}-1\right)}{(2-2 \beta-\alpha)\left(M^{2-\alpha}-1\right)}$.

The wavenumber $k$ for the rotationless limiting case can then be determined:

$k=\zeta k_{\mathrm{J}}$,

where

$k_{\mathrm{J}}=\frac{2 \pi G \mu_{0}}{\sigma_{0 \mathrm{r}}^{2}}$

is Jeans' wave number, $\zeta$ is given by

$\zeta=\frac{(2-\alpha)^{2}\left(M^{2-2 \beta-\alpha}-1\right)\left(M^{2 \beta+2-\alpha}-1\right)}{(2-2 \beta-\alpha)(2 \beta+2-\alpha)\left(M^{2-\alpha}-1\right)^{2}}$

and we have expressed $\gamma$ in terms of $\alpha$ and $\beta$. Using relation $(20)$ we find for the equation of neutral stability

$y_{0}=\gamma \zeta \hat{\lambda}\left[1-\left(\frac{2-\alpha+2 \beta}{M^{2-\alpha+2 \beta}-1}\right) L_{\mathrm{s}}\right]$.

In this expression $\hat{\lambda}$ is the wavelength in units of Toomre's critical wavelength $\lambda_{\mathrm{T}}=2 \pi / k_{\mathrm{T}}, y_{0}=\sigma_{0 \mathrm{r}}^{2} k_{\mathrm{T}}^{2} / \kappa^{2}$ and

$L_{\mathrm{s}}=\int_{1}^{M} x^{1-\alpha+2 \beta} \mathrm{e}^{-\frac{p_{0}}{\zeta x^{2 \beta}}} I_{0}\left(\frac{p_{0}}{\zeta x^{2 \beta}}\right) \mathrm{d} x$

where $p_{0}=y_{0} / \hat{\lambda}^{2}$.

Formulae (21), (22) establish the relationship between Jeans' wavenumbers for non-rotating homogeneous and heterogeneous discs. A similar result was obtained by Volkov \& Ortega (2000) by generalizing the case, previously investigated by Grishchuk \& Zel'dovich (1981), of systems composed of a certain discrete number of sub-systems. Here it arises as a particular case of Eq. (14).

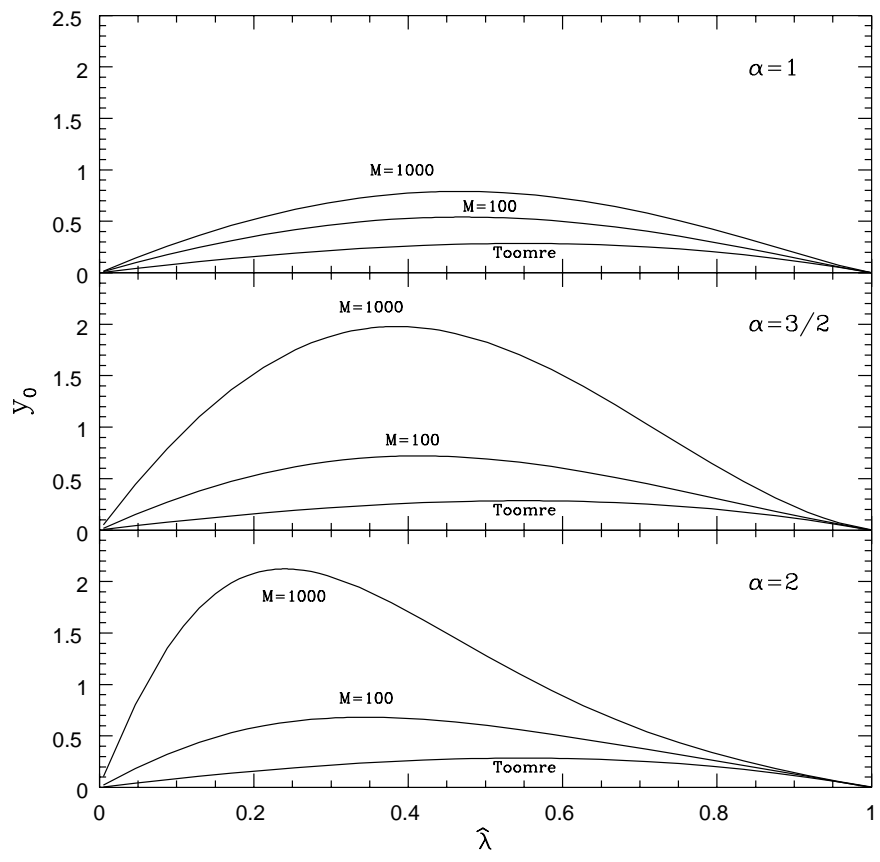

Fig. 1. Marginal stability curves for some values of the parameters $\alpha$ and $M$ with $\beta=0.5$. The lower sides of the curves denote unstable regimes

\section{Neutral stability curves and the Toomre parameter}

As shown by Toomre (1964), it is possible to compute the maximum value of the quantity $y_{0}$ from the dispersion equation. This value determines the critical velocity dispersion which stabilizes the disc against radial disturbances. We note that for $\beta=0$, that is, in the absence of any correlation between the mass and the velocity dispersion, Eq. (23) coincides with the marginal stability relation established by Toomre (1964), independent of the existence of a mass spectrum in the system. In order to explicitly show the effect of the mass spectrum when $\beta \neq 0$, we present in Fig. 1 plots of $y_{0}$ vs. $\hat{\lambda}$ for three values of the parameter $\alpha(\alpha=1.0,1.5,2.0)$. For each value of $\alpha$, marginal stability curves are plotted for $\beta=0.5$ corresponding to equipartition and for the mass spectrum ranges $M=100$ and $M=1000$. The stability proves to be very sensitive to changes in the parameter $M$ determining the mass range. We note from Fig. 1 that compared with the homogeneous (Toomre) system, the neutral stability curves have their peak values shifted in the direction of decreasing wavelengths $\hat{\lambda}$. A heterogeneous disc under the conditions investigated here is capable of becoming unstable against perturbations of smaller wavelengths $\hat{\lambda}$. In fact, it allows instability even for perturbations of wavelengths smaller than the conventional Jeans length $\lambda_{\mathrm{J}}$. This is also apparent in Fig. 2, where for more clarity we use Jeans wavelength $\hat{\lambda}_{\mathrm{J}}$ (in units of $\lambda_{\mathrm{T}}$ ) as ordinate and where we have drawn the straight lines corresponding to the limiting rotationless case with $\beta=0$ and with $\beta \neq 0$.

Toomre's parameter, defined as $Q=\sigma_{\mathrm{r}} / \sigma_{\mathrm{rc}}$, where $\sigma_{\mathrm{rc}}$ is the critical velocity dispersion, is widely employed as a 


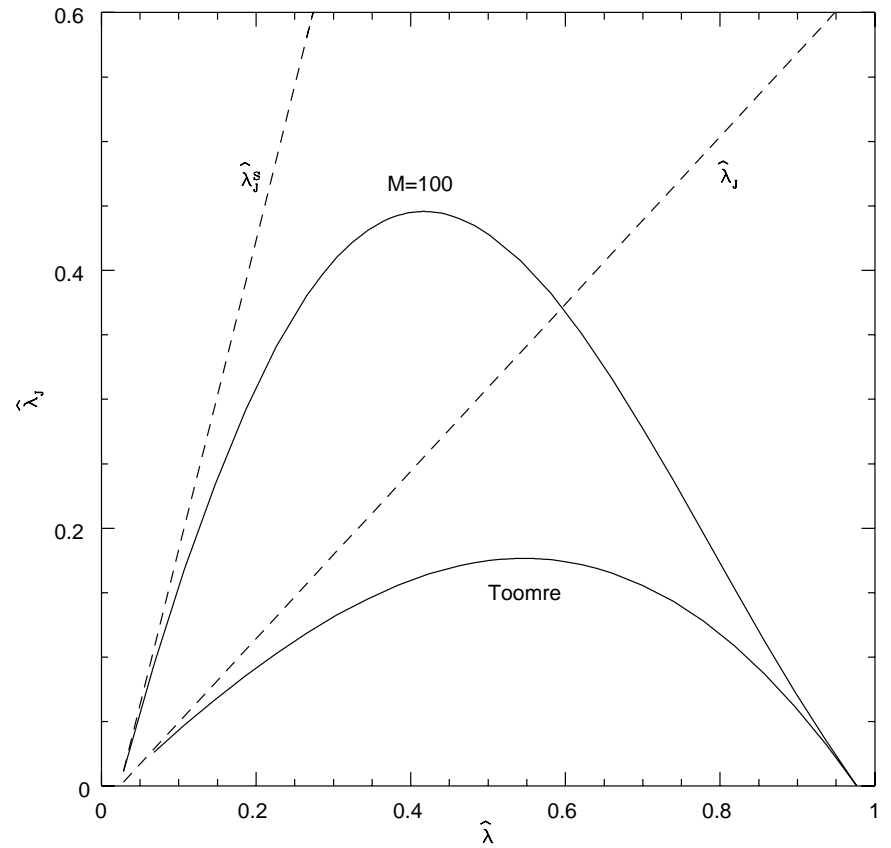

Fig. 2. Jeans' length as a function of wavelength. $\hat{\lambda}_{\mathrm{J}}^{\mathrm{s}}$ corresponds to the case without rotation with $\beta=0.5$ and $\alpha=1.5$

measure of stability in gaseous and stellar discs. From the maximum values of $y_{0}$ in the plots we can estimate the ratio $R=Q_{\mathrm{Ts}} / Q_{\mathrm{T}}$ between Toomre's parameter calculated both for $\beta \neq 0\left(\mathrm{Q}_{\mathrm{Ts}}\right)$ and for $\beta=0\left(Q_{\mathrm{T}}\right)$. In Fig. 3 we have plotted this quantity as a function of the mass spectrum range for two values of the parameter $\alpha$ using $\beta=0.5$. We notice the significant decline of this ratio with the mass spectrum range $M$.

\section{Discussion}

From the above result, we observe that for a heterogeneous thin disc with a mass spectrum and in which the random velocity and the mass of the elements are anticorrelated, the use of an average Toomre's parameter as indicator of stability may be misleading. For instance, the star formation threshold may be affected if we take the view that the onset of gravitational axisymmetric instability is a determinant of such a process (Kennicutt 1989; Chamcham et al. 1993). We note, however, that star formation is a complex process in which additional non-gravitational effects may be important and even dominant.

Our results may be of interest in studies on the dynamics of spiral galaxies where considerable structure is observed. In the case of our Galaxy, observations of the molecular clouds' kinematics (e.g., Stark 1984; Stark \& Brand 1989) have shown that the cloud velocity dispersion averaged over masses contained in a certain range yields approximately equal values in low $\left(m \lesssim 10^{4} m_{\odot}\right)$ and high $\left(m \approx 10^{5.5} m_{\odot}\right)$ mass spectrum regions. For more massive clouds $\left(m>10^{5} m_{\odot}\right)$, Stark (1983) finds that the $Z$ component of the cloud velocity dispersion decreases with mass approximately as $m^{-0.5}$. An analogous result,

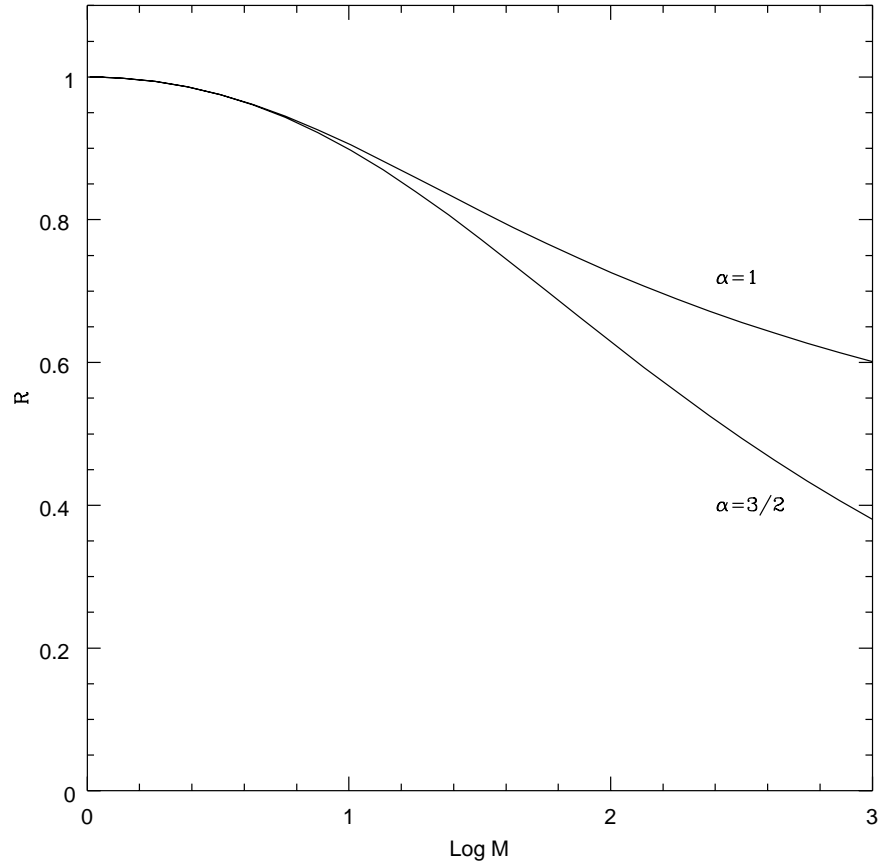

Fig. 3. Ratio of Toomre's parameter for $\beta=0$ and $\beta=0.5$ as a function of $M$ for two values of $\alpha$

consistent with equipartition, was obtained by Scoville et al. (1987) from a sample of clouds with masses within the range $\left(510^{4}-310^{6}\right) m_{\odot}$. Such a behaviour of the cloud velocity dispersion in the disc of the Galaxy has been investigated by several authors. Fukunaga (1984) and Tosa \& Fukunaga (1986) introduced gravitational viscosity, i.e. the transfer of energy from differential rotation to random motions as a heating source for molecular clouds. Considering this mechanism as the main process responsible for supporting the velocity dispersion of the clouds and equating its enery input to the losses due to inelastic collisions, Jog \& Ostriker (1988) (see also Gammie et al. 1991) obtained the equilibrium, steady-state cloud velocity dispersion. The estimated value turned out to be independent of mass and in good agreement with the observations. It has to be pointed out, however, that other equally important ingredients of the molecular cloud ensemble, such as the existence of a mass spectrum, its evolution through cloud coalescence and its effect on dissipation and shear, were not considered in these calculations. The collisional buildup of molecular clouds in the disk of the Galaxy was numerically investigated by Das \& Jog (1996). In this study, inelastic collisions, as well as the gravitational interaction between the clouds, were taken into account. Starting with a random mass distribution, a power-law mass spectrum (with $\alpha=1.7-1.9$ ) was obtained. The resulting random velocity of the clouds was $\sim 8-10 \mathrm{~km} \mathrm{~s}^{-1}$, independent of cloud mass, in good accord with the observations by Stark (1984) and Stark \& Brand (1989) in the solar vicinity ( $\lesssim 3 \mathrm{kpc}$ ). The energetics of molecular cloud systems was tackled also by Sotnikova \& Volkov (1994) on the basis of a model of selective cloud coagulation. Their solutions for the velocity 
dispersion spectrum contain two separate regions, one of constancy followed by another of abrupt fall-off for large masses. The transition depends on the initial velocity dispersion but occurs at $m \approx 10^{6} m_{\odot}$ for input values in the interval $(7.5-15) \mathrm{km} \mathrm{s}^{-1}$. If we consider the response of such a system to radial perturbations, then, according to our results, it is expected to be subject to the destabilizing effect discussed above. We here note one important characteristic of the molecular cloud ensemble in our Galaxy. The observations in the disc of the Galaxy (e.g. Solomon \& Sanders 1980) indicate that most of the mass of the molecular cloud system resides at the high end of the spectrum. This can also be estimated from our formula (8) for the surface density by computing, for fixed values of the parameter $\alpha$, the mass fraction due to clouds with masses within a given interval. For values of $\alpha$ lying between 1.5 and 2 , we obtain that in average $\simeq 80 \%$ of the total mass corresponds to clouds with masses in the range $\left(10^{5}-10^{7}\right) m_{\odot}$. It is possible that this kind of instability is responsible for the formation of the large gas concentrations with masses $m \gtrsim 10^{7} m_{\odot}$ in our Galaxy and also in other disc galaxies where giant molecular associations have been observed (e.g. Rand \& Kulkarni 1989).

\section{Conclusion}

Using the kinetic approach we have investigated the stability conditions in a flat, nonmagnetic differentially rotating disc. The disc is assumed to be made up of an ensemble of particles possessing a certain mass spectrum and obeying a specific law between the velocity dispersion and mass. In the present paper it is assumed that the velocity dispersion and the mass are related through the power-law given by Eq. (2). We have assumed that the velocity dispersion of the particles is small compared to the equilibrium rotational velocity of the motion around the centre of the disc, thus, it is valid to apply the epicyclic approximation. We consider the case where the unperturbed velocity distribution is of the form described by expression (1) which is a simple generalization of the usual Schwarzschild velocity distribution, frequently used in dynamical studies of disc configurations. Having so defined the disc configuration, we considered the reaction of this system to axisymmetric disturbances in the short-wave approximation. Of prime importance in this kind of problem is the analysis of the marginal states. Marginal stability curves were obtained from the general dispersion equation for time-independent radial perturbations. Different values of the parameters $M, \alpha$ and $\beta$ were used in order to ascertain their influence upon the stability of the system. Our choice of the values $M=100$ and $M=1000$ was motivated by the fact that most giant molecular clouds are observed to be comprised in the mass range $\left(10^{4}-10^{7}\right) m_{\odot}$. As for the parameter $\beta$, we have employed for this quantity the value $\beta=0.5$. Although other values of $\beta$, greater than 0.5 , appear to be possible in the numerical work of Sotnikova \& Volkov (1994), we have used the equipartition value because of its clear and simple physical meaning. Our results lead to the conclusion that the neutral stability curves are quite sensitive to variations in the mass spectrum range $M$ and of the parameter $\beta$. As shown in Figs. 1 and 3 the effect is already quite significant for $\beta=0.5$, especially if $M \geq 100$.

In conclusion our analysis has shown that:

a) Enhanced instability may be produced in certain regions of the mass spectrum. A somewhat more general treatment of this problem would be to find solutions for the dispersion equation allowing the parameter $\beta$ to vary with the mass spectrum;

b) The instability can take place in smaller scales as compared to the homogeneous case;

c) Toomre's stability parameter can be significantly decreased. This point suggests that the gravitational effect investigated here may be able to affect considerably the evolution of the interstellar medium in disc galaxies;

d) Our results are consistent with those obtained in Volkov \& Ortega (2000) where the hydrodynamic approach was used. Nevertheless, we think that, in the present case, the kinetic method offers a more direct way to obtain the main quantities which characterize the stability conditions of the system.

Acknowledgements. One of the authors (EV) thanks $\mathrm{CNPq}$ (Brazil) for financial support under the contract No. 301632195-2(RE). EV also acknowledges partial support from the Russian Basic Research (project 99-02-17087).

\section{References}

Chamcham, K., Pitts, E., \& Tayler, R. J. 1993, MNRAS, 263, 967

Das, M., \& Jog, C. J. 1996, ApJ, 462, 309

Gammie, Ch., F., Ostriker, J. P., \& Jog, C. J. 1991, ApJ, 378, 565

Grishchuk, L. P., \& Zel'dovich, Ya. B. 1981, Sov. Astron., 25, 3

Jog, C. J., \& Solomon, P. M. 1984, ApJ, 276, 127

Jog, C. J., \& Ostriker, J. P. 1988, ApJ, 328, 404

Jog, C. J. 1996, MNRAS, 278, 209

Kennicutt, R. C. 1989, ApJ, 344, 685

Lin, C. C., Yuan, C., \& Shu, F. 1969, ApJ, 155, 721

Polyachenko, V. L., \& Fridman, A. M. 1981, Sov. Phys., JETF, 54,1

Rand, R. J., \& Kulkarni, S. R. 1990, ApJ, 349, L43

Scoville, N. Z., Clemens, D. P., Sanders, D. B., \& Waller, W. H. 1987, ApJS, 63, 821

Solomon, P. M., \& Sanders, D. B. 1980, in Third Gregynog Symposium, Giant Molecular Clouds in the Galaxy, ed. P. M. Solomon, \& M. Edmunds (Oxford: Pergamon), 41

Sotnikova, N., \& Volkov, E. 1994, A\&A, 288, 942

Stark, A. A. 1983, in Kinematics, Dynamics and Structure of the Milky Way, ed. W. L. H. Shuter (D. Reidel Publishing Company), 127

Stark, A. A. 1984, ApJ, 281, 624

Toomre, A. 1964, ApJ, 139, 1217

Volkov, E., \& Ortega, V. G. 2000, MNRAS, 313, 112 